\title{
A NEW METHOD FOR THE CONSTRUCTION OF SOLUTIONS OF NONLINEAR WAVE EQUATIONS
}

\author{
A. F. Barannik ${ }^{1}$ and I. I. Yurik ${ }^{2}$ \\ UDC 517.9:519.46
}

We propose a simple new method for the construction of solutions of multidimensional nonlinear wave equations.

\section{Introduction}

One efficient method for the construction of solutions of nonlinear equations of mathematical physics is the method of symmetry reduction to equations with a smaller number of variables, in particular, to ordinary differential equations [1-3]. This method is based on the investigation of the subgroup structure of the invariance group of the given differential equation. The solutions thus obtained are invariant with respect to a subgroup of the invariance group of the equation. It should be noted that the invariance imposes very strict restrictions on solutions. Therefore, in many cases, the symmetry reduction does not allow one to obtain sufficiently broad classes of solutions.

In [3-9], the idea of conditional invariance of differential equations was proposed. The conditional invariance is understood as the symmetry of a certain subset of solutions. Many important nonlinear equations of mathematical physics have subsets of solutions whose symmetry substantially differs from that of the entire set of solutions. Such subsets are usually specified by additional conditions, which are certain partial differential equations. The explicit description of these additional conditions is a complicated problem and, unfortunately, there are no efficient methods for its solution.

In the present paper, we suggest a constructive method for finding certain classes of exact solutions of multidimensional nonlinear wave equations. The idea of the method is as follows: Consider a partial differential equation

$$
F\left(x, u, \frac{u}{2}, \ldots, u\right)=0
$$

where $u=u(x), x=\left(x_{0}, x_{1}, \ldots, x_{n}\right) \in R_{1, n}$, and $\underset{m}{u}$ is the collection of all possible $m$ th-order derivatives. We assume that Eq. (1) has a nontrivial symmetry algebra. To construct solutions of Eq. (1), we use a symmetry (or conditional-symmetry) ansatz [3]. We assume that it has the form

$$
u=f(x) \varphi\left(\omega_{1}, \ldots, \omega_{k}\right)+g(x)
$$

where $\omega_{1}=\omega_{1}\left(x_{0}, x_{1}, \ldots, x_{k}\right), \ldots, \omega_{k}=\omega_{k}\left(x_{0}, x_{1}, \ldots, x_{k}\right)$ are new independent variables. Ansatz (2) selects a certain subset $S$ of the entire set of solutions of Eq. (1). Let us construct, if possible, a new ansatz

$$
u=f(x) \varphi\left(\omega_{1}, \ldots, \omega_{k}, \omega_{k+1}, \ldots, \omega_{L}\right)+g(x)
$$

which is a generalization of ansatz (2). Here, $\omega_{k+1}, \ldots, \omega_{L}$ are new variables to be determined. We determine the variables $\omega_{k+1}, \ldots, \omega_{L}$ from the condition that the reduced equation corresponding to ansatz (3) coincides with the reduced equation corresponding to ansatz (2). Ansatz (3) selects a subset $S_{1}$ of the set of solutions of Eq. (1),

${ }^{1}$ Institute of Mathematics, Pedagogic Institute, Slupsk, Poland.

${ }^{2}$ State University for Food Technology, Kiev.

Translated from Ukrainskii Matematicheskii Zhumal, Vol. 51, No. 5, pp. 583-593, May, 1999. Original article submitted October 21, 1997; revision submitted February 26, 1998. 
which is an extension of the subset $S$. If the solutions belonging to the subset $S$ are known, then one can construct the solutions from the subset $S_{1}$. These solutions are constructed as follows: Let $u=u\left(x, C_{1}, \ldots, C_{t}\right)$ be a multiparameter family of solutions of Eq. (1) that have the form (2); here, $C_{1}, \ldots, C_{t}$ are arbitrary constants. Then we obtain a more general family of solutions of Eq. (1) if the constants $C_{i}$ in the solution $u=u\left(x, C_{1}, \ldots, C_{t}\right)$ are regarded as arbitrary smooth functions of $\omega_{k+1}, \ldots, \omega_{L}$.

We note that the idea of this method was formulated in [7] and developed in [8,9]. In order to apply this method to finding exact solutions of nonlinear equations of mathematical physics, one should use ansatzes (2), an algorithm for the construction of which is not indicated in [7]. In the present paper, which is a logical continuation of [7-9], the method indicated is realized for the nonlinear d'Alembert and eikonal equations as well as for Schrödinger-type equations. By using the subgroup structure of the invariance groups of the equations considered [10-12], we obtain efficient ansatzes that allow one to construct broad new classes of exact solutions containing arbitrary functions.

\section{Nonlinear d'Alembert Equation}

We consider a nonlinear Poincaré-invariant d'Alembert equation

$$
\square u+F(u)=0,
$$

where

$$
\square u=\frac{\partial^{2} u}{\partial x_{0}^{2}}-\frac{\partial^{2} u}{\partial x_{1}^{2}}-\ldots-\frac{\partial^{2} u}{\partial x_{n}^{2}}
$$

and $F(u)$ is an arbitrary smooth function. The construction of exact solutions of Eq. (4) with various conditions imposed on the function $F(u)$ is considered in [3,11-13]. The majority of these solutions are invariant with respect to the invariance group of Eq. (4), i.e., they are Lie solutions. One of the methods for the construction of solutions is the method of symmetry reduction of Eq. (4) to ordinary differential equations. The idea of this method for Eq. (4) is as follows.

Equation (4) is invariant with respect to the Poincaré algebra $A P(1, n)$ with basis elements

$$
\begin{gathered}
J_{0 a}=x_{0} \partial_{a}+x_{a} \partial_{0}, \quad J_{a b}=x_{b} \partial_{a}-x_{a} \partial_{b}, \\
P_{0}=\partial_{0}, \quad P_{a}=\partial_{a}, \quad a, b=1,2, \ldots, n .
\end{gathered}
$$

Let $L$ be an arbitrary subalgebra of rank $n$ of the algebra $A P(1, n)$. The subalgebra $L$ has two basic invariants $u$ and $\omega=\omega\left(x_{0}, x_{1}, \ldots, x_{n}\right)$. The ansatz $u=\varphi(\omega)$ that corresponds to the subalgebra $L$ reduces Eq. (4) to the ordinary differential equation

$$
\begin{gathered}
\ddot{\varphi}(\nabla \omega)^{2}+\dot{\varphi} \square \omega+F(\varphi)=0 \\
(\nabla \omega)^{2}=\left(\frac{\partial \omega}{\partial x_{0}}\right)^{2}-\left(\frac{\partial \omega}{\partial x_{1}}\right)^{2}-\ldots-\left(\frac{\partial \omega}{\partial x_{n}}\right)^{2}
\end{gathered}
$$

Such a reduction (as well as the corresponding ansatz) is called a symmetry reduction. There are eight types of nonequivalent subalgebras of rank $n$ in the algebra $A P(1, n)[11]$. 
In [14], the following procedure for the reduction of Eq. (4) to ordinary differential equations, which is a generalization of the method of symmetry reduction, was suggested: By using an ansatz $u=\varphi(\omega)$, where $\omega=\omega(x)$ is a new variable, Eq. (4) is reduced to an ordinary differential equation if $\omega(x)$ satisfies the equations

$$
\begin{gathered}
\square \omega=F_{1}(\omega), \\
(\nabla \omega)^{2}=F_{2}(\omega) .
\end{gathered}
$$

Here, $F_{1}$ and $F_{2}$ are arbitrary smooth functions that depend only on $\omega$.

Thus, having constructed all solutions of system (6), one obtains the collection of variables $\omega$ for which the ansatz $u=\varphi(\omega)$ reduces Eq. (4) to an ordinary differential equation for $\omega$. System (6) is studied in $[4,15]$.

However, it should be noted that the ansatzes obtained from system (6) do not exhaust all ansatzes that reduce Eq. (4) to ordinary differential equations. In this connection, we now consider the problem of finding generalized ansatzes (3) by using given symmetry ansatzes (2) of Eq. (4).

2.1. Consider a symmetry ansatz $u=\varphi\left(\omega_{1}\right)$ for Eq. (4), where $\omega_{1}=\left(x_{0}^{2}-x_{1}^{2}-\ldots-x_{k}^{2}\right)^{1 / 2}, k \geq 2$. This ansatz reduces Eq. (4) to the equation

$$
\varphi_{11}+\frac{k}{\omega_{1}} \varphi_{1}+F(\varphi)=0
$$

where $\varphi_{11}=\frac{d^{2} \varphi}{d \omega_{1}^{2}}, \varphi_{1}=\frac{d \varphi}{d \omega_{1}}$. This ansatz should be regarded as a particular case of the more general ansatz $u=$ $\varphi\left(\omega_{1}, \omega_{2}\right)$, where $\omega_{2}$ is an unknown variable. The ansatz $u=\varphi\left(\omega_{1}, \omega_{2}\right)$ reduces the equation

$$
\begin{gathered}
\varphi_{11}+\frac{k}{\omega_{1}} \varphi_{1}+2 \varphi_{12}\left(\nabla \omega_{1} \cdot \nabla \omega_{2}\right)+\varphi_{2} \square \omega_{2}+\varphi_{22}\left(\nabla \omega_{2}\right)^{2}+F(\varphi)=0, \\
\nabla \omega_{1} \cdot \nabla \omega_{2}=\frac{\partial \omega_{1}}{\partial x_{0}} \frac{\partial \omega_{2}}{\partial x_{0}}-\frac{\partial \omega_{1}}{\partial x_{1}} \frac{\partial \omega_{2}}{\partial x_{1}}-\ldots-\frac{\partial \omega_{1}}{\partial x_{n}} \frac{\partial \omega_{2}}{\partial x_{n}} .
\end{gathered}
$$

On Eq. (8), we impose the condition that it should coincide with the reduced equation (7). Under this assumption, Eq. (8) splits into the following two equations:

$$
\begin{gathered}
\varphi_{11}+\frac{k}{\omega_{1}} \varphi_{1}+F(\varphi)=0, \\
2 \varphi_{12}\left(\nabla \omega_{1} \cdot \nabla \omega_{2}\right)+\varphi_{22}\left(\nabla \omega_{2}\right)^{2}+\varphi_{2} \square \omega_{2}=0 .
\end{gathered}
$$

Equation (10) is satisfied for an arbitrary function $\varphi$ if the variable $\omega_{2}$ is such that

$$
\begin{gathered}
\square \omega_{2}=0, \quad\left(\nabla \omega_{2}\right)^{2}=0, \\
\nabla \omega_{1} \cdot \nabla \omega_{2}=0 .
\end{gathered}
$$

Thus, if the variable $\omega_{2}$ is chosen so that conditions (11) and (12) are satisfied, the multidimensional equation (4) reduces to the ordinary differential equation (7), and its solutions give solutions of Eq. (4). The problem of reduction thus turns into the problem of construction of general or partial solutions of system (11), (12). 
The overdetermined system (11) is studied in detail in [16, 17], where a broad class of solutions of system (11) is obtained. These solutions are constructed as follows: We consider the linear algebraic equation for the variables $x_{0}, x_{1}, \ldots, x_{n}$ with coefficients depending on the unknown $\omega_{2}$ :

$$
a_{0}\left(\omega_{2}\right) x_{0}-a_{1}\left(\omega_{2}\right) x_{1}-\ldots-a_{n}\left(\omega_{2}\right) x_{n}-b\left(\omega_{2}\right)=0
$$

Let the coefficients of this equation be analytic functions of $\omega_{2}$ satisfying the condition

$$
\left[a_{0}\left(\omega_{2}\right)\right]^{2}-\left[a_{1}\left(\omega_{2}\right)\right]^{2}-\ldots-\left[a_{n}\left(\omega_{2}\right)\right]^{2}=0
$$

Assume that Eq. (13) is solvable with respect to $\omega_{2}$ and the result of its solution is a certain function

$$
\omega_{2}\left(x_{0}, x_{1}, \ldots, x_{n}\right)
$$

which may be real or complex. Then, function (14) is a solution of system (11). We now select the solutions of (14) that have the additional property $\nabla \omega_{1} \nabla \omega_{2}=0$. It is obvious that

$$
\frac{\partial \omega_{2}}{\partial x_{0}}=-\frac{a_{0}}{\delta^{\prime}}, \quad \frac{\partial \omega_{2}}{\partial x_{1}}=\frac{a_{1}}{\delta^{\prime}}, \ldots, \frac{\partial \omega_{2}}{\partial x_{n}}=\frac{a_{n}}{\delta^{\prime}}
$$

where $\delta\left(\omega_{2}\right) \equiv a_{0}\left(\omega_{2}\right) x_{0}-a_{1}\left(\omega_{2}\right) x_{1}-\ldots-a_{n}\left(\omega_{2}\right) x_{n}-b\left(\omega_{2}\right)$ and $\delta^{\prime}$ is the derivative of $\delta$ with respect to $\omega_{2}$. Since

$$
\frac{\partial \omega_{1}}{\partial x_{0}}=\frac{x_{0}}{\omega_{1}}, \quad \frac{\partial \omega_{1}}{\partial x_{1}}=-\frac{x_{1}}{\omega_{1}}, \ldots, \quad \frac{\partial \omega_{1}}{\partial x_{n}}=-\frac{x_{n}}{\omega_{1}},
$$

we have

$$
\nabla \omega_{1} \cdot \nabla \omega_{2}=-\frac{1}{\omega_{1} \delta^{\prime}}\left(a_{0} x_{0}-a_{1} x_{1}-\ldots-a_{n} x_{n}\right)
$$

In view of (13), the equality $\nabla \omega_{1} \cdot \nabla \omega_{2}=0$ holds if and only if $b\left(\omega_{2}\right)=0$. Thus, we have constructed a broad class of ansatzes that reduce the d'Alembert equation to ordinary differential equations. The arbitrariness of the choice of the function $\omega_{2}$ may be used to satisfy certain additional conditions.

2.2. The symmetry ansatz $u=\varphi\left(\omega_{1}\right), \omega_{1}=\left(x_{1}^{2}+\ldots+x_{L}^{2}\right)^{1 / 2}, L \geq 1, L<n-1$, is generalized as follows: Let $\omega_{2}$ be an arbitrary solution of the system of equations

$$
\begin{gathered}
\frac{\partial^{2} \omega}{\partial x_{0}^{2}}-\frac{\partial^{2} \omega}{\partial x_{L+1}^{2}}-\ldots-\frac{\partial^{2} \omega}{\partial x_{n}^{2}}=0 \\
\left(\frac{\partial \omega}{\partial x_{0}}\right)^{2}-\left(\frac{\partial \omega}{\partial x_{L+1}}\right)^{2}-\ldots-\left(\frac{\partial \omega}{\partial x_{n}}\right)^{2}=0 .
\end{gathered}
$$

The ansatz $u=\varphi\left(\omega_{1}, \omega_{2}\right)$ reduces Eq. (4) to the equation 


$$
-\frac{\partial^{2} \varphi}{\partial \omega_{1}^{2}}-\frac{L-1}{\omega_{1}} \frac{\partial \varphi}{\partial \omega_{1}}+F(\varphi)=0 .
$$

If $L=n-1$, then the ansatz $u=\varphi\left(\omega_{1}, \omega_{2}\right), \omega_{2}=x_{0}-x_{n}$, is a generalization of the symmetry ansatz $u=$ $\varphi\left(\omega_{1}\right)$.

The ansatzes corresponding to subalgebras 2, 6, and 8 in Table 1 in [9] are particular cases of the ansatz constructed above. Similarly, one can obtain broad classes of ansatzes that reduce Eq. (4) to at least two-dimensional equations. Let us now present some of them.

2.3. The ansatz $u=\varphi\left(\omega_{1}, \ldots, \omega_{L}, \omega_{L+1}\right)$, where $\omega_{1}=x_{1}, \ldots, \omega_{L}=x_{L}, \omega_{L+1}$ is an arbitrary solution of system (15) and $L \leq n-1$, is a generalization of the symmetry ansatz $u=\varphi\left(\omega_{1}, \ldots, \omega_{L}\right)$ and reduces Eq. (4) to the equation

$$
-\frac{\partial^{2} \varphi}{\partial \omega_{1}^{2}}-\frac{\partial^{2} \varphi}{\partial \omega_{2}^{2}}-\ldots-\frac{\partial^{2} \varphi}{\partial \omega_{L}^{2}}+F(\varphi)=0
$$

2.4. Let $\omega_{1}=\left(x_{1}^{2}-x_{2}^{2}-\ldots-x_{L}^{2}\right)^{1 / 2}, \omega_{2}=x_{L+1} \ldots, \omega_{s}=x_{L+s-1}, L \geq 2, L+s-1 \leq n, \omega_{s+1}$ be an arbitrary solution of the system

$$
\begin{gathered}
\square \omega_{s+1}=0, \quad\left(\nabla \omega_{s+1}\right)^{2}=0, \\
\nabla \omega_{i} \cdot \nabla \omega_{s+1}=0, \quad i=1,2, \ldots, s .
\end{gathered}
$$

Then the ansatz $u=\varphi\left(\omega_{1}, \ldots, \omega_{s}, \omega_{s+1}\right)$ is a generalization of the symmetry ansatz $u=\varphi\left(\omega_{1}, \ldots, \omega_{s}\right)$ that reduces Eq. (4) to the equation

$$
\varphi_{11}-\frac{L}{\omega_{1}} \varphi_{1}-\varphi_{22}-\ldots-\varphi_{s s}+F(\varphi)=0
$$

\section{Exact Solutions of the Nonlinear d'Alembert Equation}

Let us construct some classes of exact solutions of the equation

$$
\square u+\lambda u^{k}=0, \quad k \neq 1
$$

Consider the invariant solution [12] of Eq. (17):

$$
\begin{gathered}
u^{1-k}=\sigma(k, L)\left(x_{1}^{2}+\ldots+x_{L}^{2}\right), \\
\sigma(k, L)=\frac{\lambda(1-k)^{2}}{2(L-L k+2 k)}, \quad L=1,2, \ldots, n .
\end{gathered}
$$

Applying the group transformation to solution (18), we obtain the multiparameter family of solutions

$$
u^{1-k}=\sigma(k, L)\left[\left(x_{1}+C_{1}\right)^{2}+\ldots+\left(x_{L}+C_{L}\right)^{2}\right]
$$


where $C_{1}, \ldots, C_{L}$ are arbitrary constants. Hence, according to Sec. 2.3, for $L \leq n-1$ we obtain the following family of solutions of Eq. (17):

$$
u^{1-k}=\sigma(k, L)\left[\left(x_{1}+h_{1}(\omega)\right)^{2}+\ldots+\left(x_{L}+h_{L}(\omega)\right)^{2}\right], \quad k \neq \frac{L}{L-2}
$$

where $\omega$ is an arbitrary solution of system $(15)$ and $h_{1}(\omega), \ldots, h_{L}(\omega)$ are arbitrary twice-differentiable functions of $\omega$. If, in particular, $n=3$ and $L=1$, then Eq. (17) has the following family of solutions in the space $R_{1,3}$ :

$$
u^{1-k}=\frac{\lambda(L-k)^{2}}{2(1+k)}\left[x_{1}+h_{1}(\omega)\right]^{2}, \quad k \neq-1
$$

Consider the following solution [12] of Eq. (17):

$$
\begin{gathered}
u^{1-k}=\sigma(k, s)\left(x_{0}^{2}-x_{1}^{2}-\ldots-x_{s}^{2}\right), \quad s=2, \ldots, n, \\
\sigma(k, s)=-\frac{\lambda(1-k)^{2}}{2(s-k s+k+1)}, \quad k \neq \frac{s+1}{s-1} .
\end{gathered}
$$

Solution (19) determines the multiparameter family of solutions

$$
u^{1-k}=\sigma(k, s)\left[x_{0}^{2}-x_{1}^{2}-x_{L}^{2}-\left(x_{L+1}+C_{L+1}\right)^{2}-\ldots-\left(x_{s}+C_{s}\right)^{2}\right]
$$

where $C_{L+1}, \ldots, C_{s}$ are arbitrary constants. Hence, according to Sec. 2.4, for $L \geq 2$ we obtain the family of solutions

$$
u^{1-k}=\sigma(k, s)\left[x_{0}^{2}-x_{1}^{2}-x_{L}^{2}-\left(x_{L+1}+h_{L+1}(\omega)\right)^{2}-\ldots-\left(x_{s}+h_{s}(\omega)\right)^{2}\right]
$$

where $\omega$ is an arbitrary solution of system $(16)$ and $h_{L+1}(\omega), \ldots, h_{s}(\omega)$ are arbitrary twice-differentiable functions of $\omega$. If, in particular, $L=2$ and $s=3$, then Eq. (17) has the following family of solutions in the space $R_{1,3}$ :

$$
u^{1-k}=\frac{\lambda(L-k)^{2}}{4(k-2)}\left[x_{0}^{2}-x_{1}^{2}-x_{2}^{2}-\left(x_{3}+h_{3}(\omega)\right)^{2}\right], \quad k \neq 2
$$

The equation

$$
\square u+6 u^{2}=0
$$

has the solution $u=P\left(x_{3}+C_{2}\right)$, where $P\left(x_{3}+C_{2}\right)$ is the Weierstrass elliptic function with invariants $g_{1}=0$ and $g_{3}=C_{1}$. Then, according to Sec. 2.3, we obtain the following family of solutions of Eq. (20):

$$
u=P\left(x_{3}+h(\omega)\right)
$$

Here, $\omega$ is an arbitrary solution of system (15) and $h(\omega)$ is an arbitrary twice-differentiable function. 
Consider the particular case of Eq. (17) where $k=3$. The ansatz $u=\varphi(\omega), \omega=\omega\left(x_{1}^{2}+x_{2}^{2}+x_{3}^{2}-x_{0}^{2}\right)^{1 / 2}$ reduces Eq. (17) to the equation

$$
\frac{d^{2} \varphi}{d \omega^{2}}+\frac{3}{\omega} \frac{d \varphi}{d \omega}+\lambda \varphi^{3}=0
$$

The exact solutions of Eq. (21) are constructed in [18]. We consider, e.g., the following family of solutions of Eq. (21):

$$
\varphi=\frac{1}{a \omega} \tan \left( \pm \frac{\sqrt{2}}{a^{2}} \ln \left(C_{1} \omega\right)\right), \quad \lambda=-a^{2}<0
$$

If $C_{1}$ is an arbitrary twice-differentiable function $h_{1}\left(\omega_{1}\right)$, where $\omega_{1}$ is an arbitrary solution of the system

$$
\begin{gathered}
\square \omega_{1}=0, \quad\left(\nabla \omega_{1}\right)^{2}=0, \\
\nabla \omega \cdot \nabla \omega_{1}=0,
\end{gathered}
$$

then we obtain the following family of solutions of Eq. (17):

$$
u=\frac{1}{a \omega} \tan \left( \pm \frac{\sqrt{2}}{a^{2}} \ln \left(h_{1}\left(\omega_{1}\right) \omega\right)\right) .
$$

One more family of exact solutions of Eq. (17) with $k=3$ can be obtained as follows: Applying the group transformation to solution (22), we obtain the family of solutions

$$
u=\frac{1}{a}\left[x_{1}^{2}+x_{2}^{2}+\left(x_{3}+C_{1}\right)^{2}-x_{0}^{2}\right]^{-1} \tan \left( \pm \frac{\sqrt{2}}{a^{2}} \ln C_{2}\left[x_{1}^{2}+x_{2}^{2}+\left(x_{3}+C_{1}\right)^{2}-x_{0}^{2}\right]\right) .
$$

Replacing the constants $C_{1}$ and $C_{2}$ by arbitrary functions $h_{1}\left(\omega_{1}\right)$ and $h_{2}\left(\omega_{1}\right)$, we obtain a more general family of solutions of Eq. (17).

Consider the d'Alembert equation

$$
\frac{\partial^{2} u}{\partial x_{1}^{2}}+\frac{\partial^{2} u}{\partial x_{2}^{2}}-\frac{\partial^{2} u}{\partial x_{3}^{2}}-\frac{\partial^{2} u}{\partial x_{4}^{2}}+\lambda u^{k}=0, \quad k \neq 1,
$$

in the pseudo-Euclidean space $R_{2,2}$. The symmetry ansatz $u=\varphi\left(\omega_{1}, \omega_{2}\right), \omega_{1}=x_{1}-x_{4}, \omega_{2}=x_{1}^{2}+x_{2}^{2}-x_{3}^{2}-$ $x_{4}^{2}$, reduces Eq. (23) to the equation

$$
4 \omega_{1} \varphi_{12}+4 \omega_{2} \varphi_{22}+8 \varphi_{2}+\lambda \varphi^{k}=0
$$

The generalized ansatz has the form $u=\varphi\left(\omega_{1}, \omega_{2}, \omega_{3}\right)$, where $\omega_{3}$ is an arbitrary solution of the system

$$
\square \omega_{3}=0, \quad \nabla \omega_{3} \cdot \nabla \omega_{1}=0, \quad \nabla \omega_{3} \cdot \nabla \omega_{2}=0
$$

System (25), in particular, has the solution $\omega_{3}=\left(x_{1}-x_{4}\right)\left(x_{2}-x_{3}\right)^{-1}$ and, therefore, the ansatz $u=\varphi\left(\omega_{1}\right.$, $\omega_{2}, \omega_{3}$ ) reduces Eq. (23) to Eq. (24). Equation (24) was studied in detail in [19], where the invariance algebra of 
this equation in the Lie sense was determined and some classes of exact solutions were constructed. We use, e.g., the following exact solution of Eq. (24):

$$
\varphi^{1-k}=\frac{\lambda(k-1)^{2}}{4(k-2)}\left(\omega_{2}+C_{1} \omega_{1}\right)\left(1+C_{2} \omega_{1}^{k-2}\right)
$$

where $C_{1}$ and $C_{2}$ are arbitrary constants. Replacing the constants $C_{1}$ and $C_{2}$ by arbitrary twice-differentiable functions $h_{1}\left(\omega_{3}\right)$ and $h_{2}\left(\omega_{3}\right)$, we obtain a broad class of solutions of Eq. (23):

$$
u^{1-k}=\frac{\lambda(k-1)^{2}}{4(k-2)}\left(x_{1}^{2}+x_{2}^{2}-x_{3}^{2}-x_{4}^{2}+h_{1}\left(\omega_{3}\right)\right)\left(1+h_{2}\left(\omega_{3}\right)\left(x_{1}-x_{4}\right)^{k-2}\right) .
$$

\section{Exact Solutions of the Liouville and Sine-Gordon Equations}

Consider the Liouville equation

$$
\square u+\lambda \exp u=0
$$

The symmetry ansatz $u=\varphi\left(\omega_{1}\right), \omega_{1}=x_{3}$, reduces Eq. (26) to the equation

$$
\frac{d^{2} \varphi}{d \omega^{2}}=\lambda \exp \varphi\left(\omega_{1}\right)
$$

Integrating the last equation, we establish that $\varphi$ coincides with one of the following functions:

$$
\begin{gathered}
\ln \left\{\left(-\frac{C_{1}}{2 \lambda}\right) \sec ^{2}\left[\frac{\sqrt{-C_{1}}}{2}\left(\omega_{1}+C_{2}\right)\right]\right\}, \quad C_{1}<0, \quad \lambda>0, \quad C_{2} \in R, \\
\ln \left\{\frac{2 C_{1} C_{2} \exp \left(\sqrt{C_{1}} \omega_{1}\right)}{\lambda\left[1-C_{2} \exp \left(\sqrt{C_{1}} \omega_{1}\right)\right]}\right\}, \quad C_{1}>0, \quad \lambda C_{2}>0, \\
-\ln \left(\sqrt{\frac{\lambda}{2}} \omega_{1}+C\right)^{2} .
\end{gathered}
$$

Therefore, according to Sec. 2.3, we obtain the following family of solutions of Eq. (26):

$$
\begin{gathered}
u=\ln \left\{\left(-\frac{h_{1}(\omega)}{2 \lambda}\right) \sec ^{2}\left[\frac{\sqrt{-h_{1}(\omega)}}{2}\left(x_{3}+h_{2}(\omega)\right)\right]\right\}, \quad h_{1}(\omega)<0, \quad \lambda>0, \\
u=\ln \left\{\frac{2 h_{1}(\omega) h_{2}(\omega) \exp \left(\sqrt{h_{1}(\omega)} x_{3}\right)}{\lambda\left[1-h_{2}(\omega) \exp \left(\sqrt{h_{1}(\omega)} x_{3}\right)\right]}, \quad h_{1}(\omega)>0, \quad \lambda h_{2}(\omega)>0,\right. \\
u=-\ln \left(\sqrt{\frac{\lambda}{2}} x_{3}+h(\omega)\right)^{2},
\end{gathered}
$$

where $h_{1}(\omega), h_{2}(\omega)$, and $h(\omega)$ are arbitrary twice-differentiable functions and $\omega$ is an arbitrary solution of system (15). 
By using, e.g., the solution [12] of the Liouville equation (26)

$$
u=\ln \frac{2(s-2)}{\lambda\left[x_{0}^{2}-x_{1}^{2}-\ldots-x_{s}^{2}\right]}, \quad s \neq 2
$$

we find the following broad class of solutions of this equation:

$$
u=\ln \frac{2(s-2)}{\lambda\left[x_{0}^{2}-x_{1}^{2}-\ldots-x_{L}^{2}-\left(x_{L+1}+h_{L+1}(\omega)\right)^{2}-\ldots-\left(x_{s}+h_{s}(\omega)\right)^{2}\right]},
$$

where $\omega$ is an arbitrary solution of system $(16)$ and $h_{L+1}(\omega), \ldots, h_{s}(\omega)$ are arbitrary twice-differentiable functions. If $s=3$, then Eq. (26) has the following family of solutions in the space $R_{1,3}$ :

$$
u=\ln \frac{2}{\lambda\left[x_{0}^{2}-x_{1}^{2}-x_{2}^{2}-\left(x_{3}+h_{3}(\omega)\right)^{2}\right]}
$$

By analogy, for the sine-Gordon equation $\square u+\sin u=0$, we obtain the solutions

$$
\begin{aligned}
& u=4 \arctan h_{1}(\omega) e^{\varepsilon_{0} x_{3}}-\frac{1}{2}(1-\varepsilon) \pi, \quad \varepsilon_{0}= \pm 1, \quad \varepsilon= \pm 1, \\
& u=2 \arccos \left[\ln \left(x_{3}+h_{1}(\omega), m\right)\right]+\frac{1}{2}(1+\varepsilon) \pi, \quad 0 \leq m \leq 1, \\
& u=2 \arccos \left[c n\left(\frac{x_{3}+h_{1}(\omega)}{m}, m\right)\right]+\frac{1}{2}(1+\varepsilon) \pi, \quad 0 \leq m \leq 1,
\end{aligned}
$$

where $h_{1}(\omega)$ is an arbitrary twice-differentiable function and $\omega$ is an arbitrary solution of system (15).

\section{Exact Solutions of the Eikonal Equation}

We consider the eikonal equation

$$
\left(\frac{\partial u}{\partial x_{0}}\right)^{2}-\left(\frac{\partial u}{\partial x_{1}}\right)^{2}-\left(\frac{\partial u}{\partial x_{2}}\right)^{2}-\left(\frac{\partial u}{\partial x_{3}}\right)^{2}=1
$$

The symmetry ansatz $u=\varphi\left(\omega_{1}\right), \omega_{1}=x_{0}^{2}-x_{1}^{2}-x_{2}^{2}-x_{3}^{2}$, reduces Eq. (27) to the equation

$$
4 \omega_{1}\left(\frac{\partial \varphi}{\partial \omega_{1}}\right)^{2}-1=0
$$

We seek a generalized ansatz in the form $u=\varphi\left(\omega_{1}, \omega_{2}\right)$. This ansatz reduces Eq. (27) to the equation

$$
4 \omega_{1}\left(\frac{\partial \varphi}{\partial \omega_{1}}\right)^{2}+2\left(\nabla \omega_{1} \cdot \nabla \omega_{2}\right) \frac{\partial \varphi}{\partial \omega_{1}}+\left(\nabla \omega_{2}\right)^{2}\left(\frac{\partial \varphi}{\partial \omega_{2}}\right)^{2}=1
$$


Assume that Eq. (29) coincides with the reduced equation (28). It is obvious that this condition is satisfied if $\omega_{2}$ is such that

$$
\left(\nabla \omega_{2}\right)^{2}=0, \quad \nabla \omega_{1} \cdot \nabla \omega_{2}=0
$$

Solving system (30), we determine the explicit form of the variable $\omega_{2}$. It is easy to see that an arbitrary function of solution (30) is also a solution of system (30). Integrating Eq. (28), we get $(u+C)^{2}=x_{0}^{2}-x_{1}^{2}-x_{2}^{2}-x_{3}^{2}$, where $C$ is an arbitrary constant. One can obtain a more general family of solutions of the eikonal equation if $C$ is regarded as an arbitrary solution of system (30).

The symmetry ansatz $u=\varphi\left(\omega_{1}, \omega_{2}\right), \omega_{1}=x_{0}^{2}-x_{1}^{2}-x_{2}^{2}, \omega_{2}=x_{3}$, is generalized as follows: Let $\omega_{3}$ be an arbitrary solution of the system of equations

$$
\begin{gathered}
\left(\frac{\partial \omega_{3}}{\partial x_{0}}\right)^{2}-\left(\frac{\partial \omega_{3}}{\partial x_{1}}\right)^{2}-\left(\frac{\partial \omega_{3}}{\partial x_{2}}\right)^{2}=0 \\
x_{0} \frac{\partial \omega_{3}}{\partial x_{0}}+x_{1} \frac{\partial \omega_{3}}{\partial x_{1}}+x_{2} \frac{\partial \omega_{3}}{\partial x_{2}}=0 .
\end{gathered}
$$

Then the ansatz $u=\varphi\left(\omega_{1}, \omega_{2}, \omega_{3}\right)$ reduces the eikonal equation to the equation

$$
4 \omega_{1}\left(\frac{\partial \varphi}{\partial \omega_{1}}\right)^{2}-\left(\frac{\partial \varphi}{\partial \omega_{2}}\right)^{2}=1
$$

Equation (32) has the solutions [12]

$$
\begin{gathered}
\varphi=\frac{C_{1}^{2}+1}{2 C_{1}}\left(x_{0}^{2}-x_{1}^{2}-x_{2}^{2}\right)^{1 / 2}+\frac{C_{1}^{2}-1}{2 C_{1}} x_{3}+C_{2}, \\
\left(\varphi+C_{2}\right)^{2}=x_{0}^{2}-x_{1}^{2}-x_{2}^{2}-\left(x_{3}+C_{1}\right)^{2},
\end{gathered}
$$

which can easily be found by applying the method of symmetry reduction to Eq. (32), which reduces it to ordinary differential equations. Replacing the arbitrary constants $C_{1}$ and $C_{2}$ by arbitrary functions $h_{1}\left(\omega_{3}\right)$ and $h_{2}\left(\omega_{3}\right)$, we obtain the following broader classes of exact solutions of the eikonal equation:

$$
\begin{gathered}
u=\frac{h_{1}\left(\omega_{3}\right)^{2}+1}{2 h_{1}\left(\omega_{3}\right)}\left(x_{0}^{2}-x_{1}^{2}-x_{2}^{2}\right)^{1 / 2}+\frac{h_{1}\left(\omega_{3}\right)^{2}-1}{2 h_{1}\left(\omega_{3}\right)} x_{3}+h_{2}\left(\omega_{3}\right), \\
\left(u+h_{2}\left(\omega_{3}\right)\right)^{2}=x_{0}^{2}-x_{1}^{2}-x_{2}^{2}-\left(x_{3}+h_{1}\left(\omega_{3}\right)\right)^{2} .
\end{gathered}
$$

Note that we have thus also derived broad classes of exact solutions of the Born-Infeld equation because it is a differential consequence of the eikonal equation [3].

Consider the following eikonal equation:

$$
\left(\frac{\partial u}{\partial x_{0}}\right)^{2}-\left(\frac{\partial u}{\partial x_{1}}\right)^{2}-\left(\frac{\partial u}{\partial x_{2}}\right)^{2}-\left(\frac{\partial u}{\partial x_{3}}\right)^{2}=-1
$$


The symmetry ansatz $u=\varphi\left(\omega_{1}\right), \omega_{1}=x_{3}$, reduces Eq. (33) to the equation $\dot{\varphi}^{2}=1$. The reduced equation has a solution $\varphi=\varepsilon x_{3}+C$, where $\varepsilon= \pm 1$ and $C$ is an arbitrary constant. Replacing the constant $C$ by an arbitrary function $h\left(\omega_{2}\right)$, where $\omega_{2}$ is an arbitrary solution of the equation

$$
\left(\frac{\partial u}{\partial x_{0}}\right)^{2}-\left(\frac{\partial u}{\partial x_{1}}\right)^{2}-\left(\frac{\partial u}{\partial x_{2}}\right)^{2}=0
$$

we obtain a more general family of solutions of Eq. (33):

$$
u=\varepsilon x_{3}+h\left(\omega_{2}\right)
$$

\section{On Exact Solutions of a Schrödinger-Type Equation}

Consider the equation

$$
i \frac{\partial \psi}{\partial t}=k \square \psi+\psi F(|\psi|),
$$

where $\psi=\psi\left(t, x_{0}, \ldots, x_{n}\right)$ and

$$
\square \psi=\frac{\partial^{2} \psi}{\partial x_{0}^{2}}-\frac{\partial^{2} \psi}{\partial x_{1}^{2}}-\ldots-\frac{\partial^{2} \psi}{\partial x_{n}^{2}}
$$

The symmetry ansatz $\psi=\varphi(t)$ reduces Eq. (34) to the equation

$$
i \dot{\varphi}-\varphi F(|\varphi|)=0
$$

This ansatz is a particular case of a more general ansatz

$$
\psi=\varphi(t, \omega)
$$

where $\omega$ is an arbitrary solution of the system of equations

$$
\begin{gathered}
i \frac{\partial \omega}{\partial t}=k \square \psi, \\
\left.(\nabla \omega)^{2}=0\right) .
\end{gathered}
$$

Thus, formula (36) defines a family of solutions of the nonlinear multidimensional equation (34) if $\varphi$ satisfies (35) and $\omega$ is a solution of system (37).

The formula

$$
\psi=\exp \left\{-\frac{i\left(x_{0}^{1}-x_{1}^{2}-\ldots-x_{n}^{2}\right)}{4 k t}\right\} \varphi\left(\omega_{1}, \omega_{2}\right)
$$

is an ansatz for Eq. (34) if $\omega_{1}=t, \omega_{2}$ satisfies Eq. (37), and 


$$
x_{0} \frac{\partial \omega_{2}}{\partial x_{0}}+x_{1} \frac{\partial \omega_{2}}{\partial x_{1}}+\ldots+x_{n} \frac{\partial \omega_{2}}{\partial x_{n}}=0
$$

The reduced equation has the form

$$
i \frac{\partial \varphi}{\partial t}+\frac{(n+1) i}{2 t} \varphi-\varphi F(|\varphi|)=0
$$

Therefore, solving Eq. (40) and system (37), (39) and inserting the solutions obtained in formula (38), we get broad classes of exact solutions of Eq. (34).

\section{System of Nonlinear d'Alembert and Eikonal Wave Equations}

Consider the system of equations

$$
\begin{aligned}
& \square u=F(u), \\
& (\nabla u)^{2}=-1,
\end{aligned}
$$

where $u=u\left(x_{0}, x_{1}, x_{2}, x_{3}\right)$. System (41) was investigated in $[14,20]$, where it was proved, in particular, that this system is compatible if and only if $F(u)=N(u+C)^{-1}$, where $C$ is an arbitrary constant and $T$ is a discrete parameter that may take one of the values $0,1,2,3$. Let us construct broad classes of exact solutions of system (41) in the form $F(u)=0$ and $F(u)=3 u^{-1}$. It is obvious that the system

$$
\square u=0, \quad(\nabla u)^{2}=-1
$$

has an invariant solution $u=\varepsilon x_{3}+C_{1}$, where $C_{1}$ is an arbitrary constant. Therefore, according to Sec. 2.4, system (42) has the family of exact solutions $u=\varepsilon x_{3}+h_{1}(\omega)$, where the function $\omega=\omega\left(x_{0}, x_{1}, x_{2}\right)$ is an arbitrary solution of the system $\square \omega=0,(\nabla \omega)^{2}=0$. The system

$$
\square u=3 u^{-1}, \quad(\nabla u)^{2}=-1
$$

has the invariant solution $u^{2}=x_{0}^{2}-x_{1}^{2}-x_{2}^{2}-x_{3}^{2}$. Applying the group transformation to this solution, we obtain the solution $u^{2}=x_{0}^{2}-x_{1}^{2}-x_{2}^{2}-\left(x_{3}+C\right)^{2}$. Thus, according to Sec. 2.4 , system (43) has the family of solutions

$$
u^{2}=x_{0}^{2}-x_{1}^{2}-x_{2}^{2}-\left(x_{3}+h(\omega)\right)^{2}
$$

where the function $\omega=\omega\left(x_{0}, x_{1}, x_{2}\right)$ is a solution of the system

$$
\begin{gathered}
\square \omega_{3}=0, \quad(\nabla \omega)^{2}=0, \\
x_{0} \frac{\partial \omega}{\partial x_{0}}+x_{1} \frac{\partial \omega}{\partial x_{1}}+x_{2} \frac{\partial \omega}{\partial x_{2}}=0 .
\end{gathered}
$$

The generalization of these results to an arbitrary number $n$ is quite obvious. 


\section{REFERENCES}

1. L. V. Ovsiannikov, Group Analysis of Differential Equations, Academic Press, New York (1982).

2. J. Olver, Applications of Lie Groups to Differential Equations, Springer, New York (1986).

3. W. I. Fushchych, V. M. Stelen, and N. I. Serov, Symmetry Analysis and Exact Solutions of Equations of Nonlinear Mathematical Physics, Kluwer, Dordrecht (1993).

4. W. I. Fushchych and A. G. Nikitin, Symmetries of Maxwell's Equations, Reidel. Dordrecht (1987).

5. W. I. Fushchych and I. M. Tsyfra, "On a reduction and solutions of nonlinear wave equations with broken symmetry," J. Phys. A: Math. Gen., 20, No. 2, L45-L48 (1987).

6. D. Levi and P. Winternitz, "Non-classical symmetry reduction-example of the Boussinesq equation," J. Phys. A, 22, No. 2, 2915-2924 (1989).

7. V. I. Fushchich and A. F. Barannik, "A new method for the construction of exact solutions of nonlinear wave equations," Dopov. Akad. Nauk Ukr., No. 10, 48-51 (1996).

8. A. F. Barannyk and I. I. Yuryk, "On some exact solutions of nonlinear wave equations," in: Proceedings of the Second International Conference "Symmetry in Nonlinear Mathematical Physics," Vol. 1 (1997), pp. 98-107.

9. A. F. Barannyk and I. I. Yuryk, "On a new method for constructing exact solutions of nonlinear differential equations of mathematical physics," J. Phys. A: Math. Gen., 31, 4899-4907 (1998).

10. A. F. Barannik and I. I. Yurik, "Classification of maximal subalgebras of rank $n$ of the conformal algebra $A C(1, n)$," $U k r$. Mat. Zh., 50, No. 4, 459-470 (1998).

11. A. M. Grunland, J. Harnad, and P. Winternitz, "Symmetry reduction of nonlinear relativistically invariant equations," J. Math. Phys., 25, No. 4, 791-806 (1984).

12. V. I. Fushchich, L. F. Barannik, and A. F. Barannik, Subgroup Analysis of Galilei and Poincaré Groups and Reduction of Nonlinear Equations [in Russian], Naukova Dumka, Kiev (1991).

13. G. Cieciura and A. Grunland, "A certain class of solutions of the nonlinear wave equations," J. Math. Phys., 25, No. 12, 3460-3469 (1984).

14. V. I. Fushchich and R. Z. Zhdanov, Nonlinear Spinor Equations: Symmetry and Exact Solutions [in Russian], Naukova Dumka, Kiev (1992).

15. C. B. Collins, "Complex potential equations. I. A technique for solutions," Math. Proc. Cambridge Philos. Soc., 80, Part 1, 165187 (1976).

16. V. I. Smimov and S. L. Sobolev, "A new method for the solution of the two-dimensional problem of elastic vibrations," Tr. Seismolog. Inst. Akad. Nauk SSSR, No. 20, 37-42 (1932).

17. V. I. Smirnov and S. L. Sobolev, "On the application of a new method to the investigation of elastic vibrations," Tr. Seismolog. Inst. Akad. Nauk SSSR, No. 29, 43-51 (1933).

18. J. Patera, R. T. Sharp, P. Winternitz, and H. Zassenhauss, "Subgroups of the Poincaré group and their invariants," J. Math. Phys., 17, No. 6, 977-985 (1976).

19. V. I. Fushchich, A. F. Barannik, and Yu. D. Moskalenko, "On new exact solutions of the multidimensional nonlinear d'Alembert equation," Dopov. Akad. Nauk Ukr., No. 2, 33-37 (1995).

20. V. I. Fushchich, R. Z. Zhdanov, and I. V. Revenko, "General solutions of a nonlinear wave equation and the eikonal equation," $U k r$. Mat. Zh., 43, No. 11, 1471-1487 (1991). 